\title{
Student recognition of interference and diffraction patterns: An eye-tracking study
}

\author{
Ana Susac $\odot,{ }^{1, *}$ Maja Planinic $\odot,{ }^{2}$ Andreja Bubic, ${ }^{3}$ Lana Ivanjek, ${ }^{4}$ and Marijan Palmovic ${ }^{5}$ \\ ${ }^{1}$ Department of Applied Physics, Faculty of Electrical Engineering and Computing, \\ University of Zagreb, Unska 3, 10000 Zagreb, Croatia \\ ${ }^{2}$ Department of Physics, Faculty of Science, University of Zagreb, Bijenicka 32, 10000 Zagreb, Croatia \\ ${ }^{3}$ Chair for Psychology, Faculty of Humanities and Social Sciences, \\ University of Split, Sinjska 2, 21000 Split, Croatia \\ ${ }^{4}$ Faculty of Physics, University of Vienna, Porzellangasse 4, 1090 Vienna, Austria \\ ${ }^{5}$ Laboratory for Psycholinguistic Research, Department of Speech and Language Pathology, \\ University of Zagreb, Borongajska cesta 83h, 10000 Zagreb, Croatia
}

(Received 22 January 2020; accepted 15 September 2020; published 11 November 2020)

\begin{abstract}
Previous studies have demonstrated that students have difficulties in applying the wave model of light to explain single-slit diffraction and double-slit interference patterns. In this study, we investigated if students could recognize typical interference and diffraction patterns at all. Eye movements of high-school students were measured while they were identifying patterns produced by monochromatic light on a double slit, single slit, and diffraction grating, and by white light on a diffraction grating. Most students had difficulties with recognizing double-slit interference pattern and diffraction grating pattern of monochromatic light. Identification of the single-slit diffraction pattern was easier probably due to its distinguishable central maximum. The easiest task for students was recognizing the diffraction pattern of white light on an optical grating. Eye-tracking data suggested that even students who incorrectly answered this question were aware that the diffraction grating separates white light into colors. Additionally, eye tracking revealed that students who identified patterns correctly attended more the correct pattern than other options, thus corroborating previous findings. Overall, the results indicate that the recognition of interference and diffraction patterns is quite demanding for students, suggesting that more attention should be paid to observing and understanding basic wave optics phenomena.
\end{abstract}

DOI: 10.1103/PhysRevPhysEducRes.16.020133

\section{INTRODUCTION}

Wave optics represents a part of physics that is usually included in high-school physics curricula as well as many university physics courses. However, studies on students' understanding of wave optics have been relatively rare and were mostly conducted on university students [1-8], whereas only a few explored high-school students' difficulties with wave optics $[9,10]$ or first-year university students who did not take university courses covering wave optics [11]. Previous studies found that most student difficulties are related to geometrical optics reasoning in contexts that require the application of the wave model $[1,4]$. A detailed list of students' difficulties in wave optics was presented in the recent paper by Mesic et al. [6].

\footnotetext{
*Corresponding author. ana.susac@fer.hr

Published by the American Physical Society under the terms of the Creative Commons Attribution 4.0 International license. Further distribution of this work must maintain attribution to the author(s) and the published article's title, journal citation, and DOI.
}

When taught within formal education curricula, wave optics is usually introduced after covering basic concepts related to mechanical waves, such as wavelength, frequency, propagation speed, etc. More advanced topics, such as interference, are also often introduced when studying mechanical waves. Previous studies reported students' difficulties with understanding the relationship between the concepts of wavelength, frequency, and wave speed at the boundary between two different media [12] and difficulties with expressing a physical distance, such as the separation between two sources, in terms of wavelength [13]. Kryjevskaia et al. suggested these difficulties could inhibit students' understanding of wave phenomena such as interference and diffraction. Interference of light waves can be described as a superposition phenomenon analogous to the superposition of mechanical waves. Basic phenomena of wave optics (interference and diffraction of light) are usually introduced through exploring how different patterns are created by the superposition of light waves on the double slit, single slit, and diffraction grating. Hence, the recognition of typical interference and diffraction patterns, such as those that can be found in textbooks, should be one of the important learning objectives in wave optics. 
Although this may appear as a rather simple and cognitively not very demanding objective, our experience suggests that both university and high-school students often confound interference and diffraction patterns obtained by different optical elements (slits, gratings). To our knowledge, there are no physics education research (PER) studies focused on this particular issue (although recognition of interference and diffraction patterns was required in some multiple-choice questions used by Mesic et al. [6]). Consequently, we decided to investigate high-school students' recognition of interference and diffraction patterns. Furthermore, we used eye tracking to obtain more information on students' visual attention during task solving.

Eye tracking is increasingly used in PER studies, mostly to explore problem solving in different areas of physics. For example, eye tracking was used to examine what students look at when studying worked-out examples in mechanics [14] or when they solve problems by themselves [15]. Eye tracking gave new insights into students' understanding of graphs [16-18] and divergence of vector fields [19]. Research findings on differences in visual attention between those who correctly and incorrectly answer problems helped researchers to develop visual cues that directed students' attention to relevant areas to facilitate problem solving [20-22]. Students' eye movements successfully predicted their answers on multiple-choice questions [23], including standard instruments, such as the Force Concept Inventory (FCI) and its representational variant R-FCI $[24,25]$. Eye tracking was also used to investigate if graphical representations of data improve student understanding of measurement [26] and to determine if supportive diagrams that visualize the physical situation help students in problem solving [27]. Given the previous successful use of eye tracking in PER research, it was also employed in this study to further explore the nature and dynamics of students' problem solving in the context of wave optics. In particular, we used multiple-choice questions like in most previous studies and wanted to compare visual attention of students who correctly and incorrectly answered the questions $[15,18,24,25,28]$ with a new type of offered answers, i.e., typical interference and diffraction patterns.

In one of the earlier studies of visual attention during solving multiple-choice science problems, Tsai et al. found that students attend more the chosen answer than the rejected options [28]. Tsai et al. analyzed data only from six students and one problem. However, recent studies using a larger student sample and the whole tests (FCI, Test of Understanding Graphs in Kinematics TUG-K), or a subset of test items from developed instruments (R-FCI), obtained similar results $[18,24,25]$. Taken together, these results suggest that students who correctly answer questions attend most the correct options. Students who incorrectly answer questions do not always have a consistent pattern of eye movements; sometimes they equally distribute attention on all options whereas in some cases they attend more one or two options, usually attending least the correct options. Tsai et al. argued that students who correctly answer questions have higher metacognitive skills and are able to recognize and concentrate on relevant factors during problem solving [28]. Some results suggested that students who correctly answer questions already know the correct answer after reading the question, so they only have to find the correct option, or sometimes they are able to quickly reject some options as irrelevant without careful consideration [25]. On the other hand, students who are not experts could be expected to distribute their attention equally on different options. Results from previous studies suggested that these students often choose naïve options [18,24].

Most of the previous PER studies using eye tracking and multiple-choice questions concentrated on mechanics $[15,18,24,25]$. We decided to extend on previous work with multiple-choice questions by investigating students' visual attention during recognition of typical patterns from wave optics. Visual stimuli used in this study were perceptually different from the graphs and pen drawing pictures used in the previous studies $[15,18,24,25]$. Since the multiplechoice answers offered in this study were novel, we wanted to examine if the distribution of students' attention corresponded to the previous findings when different options were offered (e.g., graphs, text statements, etc.).

In this study, we aim to answer the following research questions:

(i) Do students recognize and distinguish typical interference and diffraction patterns obtained by the double slit, single slit, and diffraction grating?

(ii) What is the difference in the distribution of visual attention between students who identify interference and diffraction patterns correctly and incorrectly?

Our hypothesis for the first research question was that high school students would have difficulties in recognizing typical interference and diffraction patterns. Based on the results from the previous studies, we expected that the students who identify interference and diffraction patterns correctly would attend more the correct pattern than the other options, and students who identify patterns incorrectly would attend equally all options or less the correct pattern than the other options.

\section{METHODS}

\section{A. Participants}

Participants in this study were 35 high-school students (age 18 years) in the last (fourth) year of high school. All participants attended different general education and science-mathematics types of gymnasiums in Zagreb, Croatia where physics is taught as a compulsory subject whose curriculum covers interference and diffraction of light (double-slit interference, diffraction on a single slit, 
diffraction grating). We used convenient sampling, voluntary participants who were prepared to come to university for eye-tracking measurement and to answer some physics questions. These were final-year high school students, who studied wave optics for about six weeks as a part of their regular physics instruction ( 2 or 3 hours per week, depending on the type of school). Students had observed some demonstration experiments on interference and diffraction of light, and had possibly seen pictures of double-slit, single-slit, and optical grating patterns in their textbooks. However, only about half of the participants had performed some experiments themselves (mostly double-slit and diffraction grating experiments).

\section{B. Materials}

Four multiple-choice questions on interference and diffraction patterns were used in this study. The questions were the following:

Q1: Which of the patterns on the screen was produced by the interference of light from two coherent sources? (Possible answers were four green patterns shown on the left side of Fig. 1.)

Q2: Which of the patterns on the screen was produced by the diffraction of green light on a single slit? (Possible answers were four green patterns shown on the left side of Fig. 1.)

Q3: Which of the patterns on the screen was produced by the diffraction of green light on an optical grating? (Possible answers were four green patterns shown on the left side of Fig. 1.)

Q4: Which of the patterns on the screen was produced by the diffraction of white light on an optical grating? (Possible answers were two gray and two rainbow patterns shown on the right side of Fig. 1.)

The interference and diffraction patterns were taken from the website of Dietrich Zawischa [29] and modified

Q3) Koja je od ovih siika na zastoru nastala ogibom zelene svjetlosti na optičkoj rešetki?

(a)

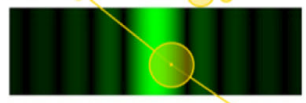

(b)

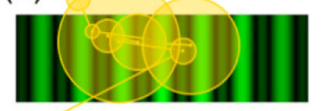

(c)

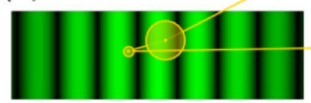

(d)

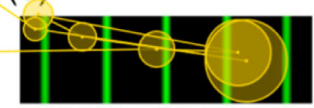

(Fig. 1). In item design, we used only those wave optics patterns that students were supposed to be familiar with. Otherwise, had students been presented with some other patterns, those would not have been the appealing options for them. The first three questions (Q1-Q3) with the same four green patterns were presented to participants in counterbalanced order, and the question Q4 was presented at the end. Thus, it is important to note that the labels of questions Q1-Q3 do not imply the order in which they were presented.

\section{Procedure}

Eye-movement data were recorded using the SMI screenbased RED-m system (SensoMotoric Instruments G.m.b.H.) with sample rate $120 \mathrm{~Hz}$ integrated with 17" TFT LCD monitor (Samsung). The participants were free to slightly move their head during the measurements. The eye-tracking system was calibrated for each participant before the data recording using a 5-point calibration algorithm. Questions were presented on a monitor at the distance of $50 \mathrm{~cm}$ from the participants' eyes. The size of each pattern on the screen was $10 \mathrm{~cm} \times 3 \mathrm{~cm}$. By choosing the answer, participants advanced to the next question. There was no time limit to answer the questions. The whole procedure, including explanation, eye-movement calibration and recording lasted around $5 \mathrm{~min}$. Since the recording was so short, there was no drift check before questions. Calibration data and visual inspection of scan paths showed a reasonably good quality of data for all participants, so all data were analyzed. Blinks were automatically detected by the software. Since the recording was short, the number of blinks was low. Further examination of the animated scan paths allowed the detection of additional periods when the eye movements were not recorded. Overall, the system recorded the eye positions during approximately $90 \%$ of time.

Q4) Koja je od ovih slika na zastoru nastala

$$
\text { ogibom bijele svjetlosti na optičkoj rešetki? }
$$

(a)

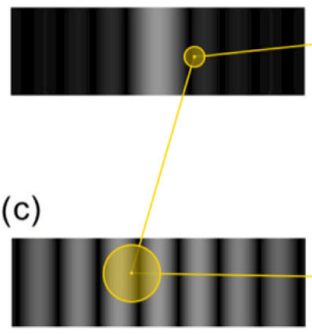

(b)

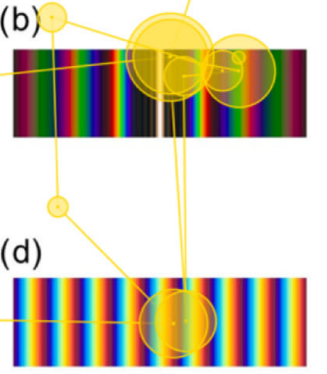

FIG. 1. Example of one participant's scan path for questions Q3 and Q4. The centers of circles show positions of fixations (when the eyes remain relatively still) and the lines show saccades (rapid eye movements between fixations). The radii of circles are proportional to the duration of fixations. The four green patterns on the left were possible answers for questions Q1, Q2, and Q3, whereas four patterns on the right were possible answers for question $\mathrm{Q} 4$. 
TABLE I. The percentages of students that selected a particular choice $(a, b, c, d)$ for each question. The correct answer is in bold.

\begin{tabular}{lcccc}
\hline \hline Question & $a$ & $b$ & $c$ & $d$ \\
\hline Q1 double slit & 34 & 23 & $\mathbf{2 0}$ & 23 \\
Q2 single slit & $\mathbf{6 3}$ & 9 & 11 & 17 \\
Q3 diffraction grating & 46 & 11 & 23 & $\mathbf{2 0}$ \\
Q4 diffraction grating (white light) & 0 & $\mathbf{8 3}$ & 6 & 11 \\
\hline \hline
\end{tabular}

\section{Data analysis}

Students' responses were scored correct or incorrect. The recorded eye movements data were analyzed using BeGaze software that allows evaluation of the eye fixations and saccades. The identification by dispersion-threshold (IDT) algorithm was used to determine fixations with maximum dispersion value $100 \mathrm{px}$ and minimum fixation duration $80 \mathrm{~ms}$. We defined five rectangular areas of interest (AOIs) for each question that included the text of the problem (question) and multiple-choice answers $(a, b, c$, and $d)$. The following eye-tracking measures were evaluated and compared for the students who correctly and incorrectly identified interference and diffraction patterns: dwell time (viewing time), number of fixations, average fixation duration, and number of revisits (returns to previously inspected AOI).

The number of students who identified patterns correctly and incorrectly was unbalanced (Table I). Given that we could not suppose that the variances were the same, we compared separately dwell times of students who identified interference and diffraction patterns correctly and incorrectly. Several one-way analyses of variance (ANOVAs) and Tukey's HSD (honestly significant difference) tests were conducted. A threshold of $p=0.05$ was used for determining the level of effect significance within all conducted tests. When performing the analyses, multiple comparisons were not conducted because, by tapping into different questions, we were mostly making independent investigations. Instead of making these which would increase the probability of type II error, we report the relevant effect sizes [30,31].

\section{RESULTS}

The percentages of students that selected a particular pattern are shown in Table I. The percentage of correct answers was the highest for question Q4, on which $83 \%$ of students correctly identified the diffraction pattern of white light on an optical grating. Furthermore, $63 \%$ of students correctly identified the single-slit diffraction pattern. Students did not perform as well on questions Q1 and Q3, in which they had to recognize double-slit interference pattern and diffraction grating pattern. Only $20 \%$ of the high-school students in our sample gave correct answers on questions Q1 and Q3.
To explore the visual attention of students who identified interference and diffraction patterns correctly and incorrectly, we compared dwell times (viewing times) for multiple-choice answers $(a, b, c$, and $d)$ for each question (Fig. 2). Table II shows the results of the corresponding one-way ANOVAs. The results of pairwise comparisons are shown in Fig. 2.

The results for questions Q1-Q3 indicate that students who identified the interference and diffraction patterns for monochromatic light (shown on the left side of the Fig. 1) correctly attended more the correct answer than the other options (Fig. 2). For question Q1, students who correctly answered the question attended more the double-slit pattern (correct answer $c$ ) than the single-slit pattern (incorrect answer $a$ ); other differences were not statistically different. For question Q2, students attended more the single-slit pattern (correct answer $a$ ) than the other three options. For question Q3, there were no statistically significant differences, but the trend was the same as in questions Q1 and Q2; students who correctly answered the question had a tendency to spend the longest time attending the correct answer $d$ (diffraction grating pattern).

For students who incorrectly identified the interference and diffraction patterns in questions Q1-Q3, there was no statistically significant difference between dwell times for multiple-choice answers $(a, b, c$, and $d)$. However, the trend was that the students who incorrectly answered the questions Q2 and Q3 appeared to spend the shortest time attending the correct answers.

The results for questions Q4 show that students who identified the pattern produced by the diffraction of white light on an optical grating correctly attended more the correct pattern than the other options (Fig. 2). Students who identified the pattern incorrectly had longer dwell times for answers $b$ and $d$ than answers $a$ and $c$.

Since eye-tracking data showed considerable interindividual differences in time that students spent attending multiple-choice options before choosing an answer, we also evaluated the proportion of dwell time on multiple-choice answers $(a, b, c$, and $d)$ for each question. The results are shown in the Supplemental Material [32] (Fig. S1). The corresponding one-way ANOVAs (Table S1 [32]) and pairwise comparisons confirm the above results with some pairwise comparisons now showing statistically significant differences. For example, the proportion of dwell time on correct answer $c$ in question Q1 is longer than the proportion of dwell time on all other answers.

Furthermore, we evaluated average fixation durations for multiple-choice answers $(a, b, c$, and $d)$ for each question. Figure 3 and Table III show the results of the corresponding one-way ANOVAs and pairwise comparisons. The duration of fixations was not variable across multiple-choice answers as the dwell time, yet we found some statistically significant differences. Students who incorrectly answered question Q1 had a longer fixation duration for answer $c$ than answer $d$. 


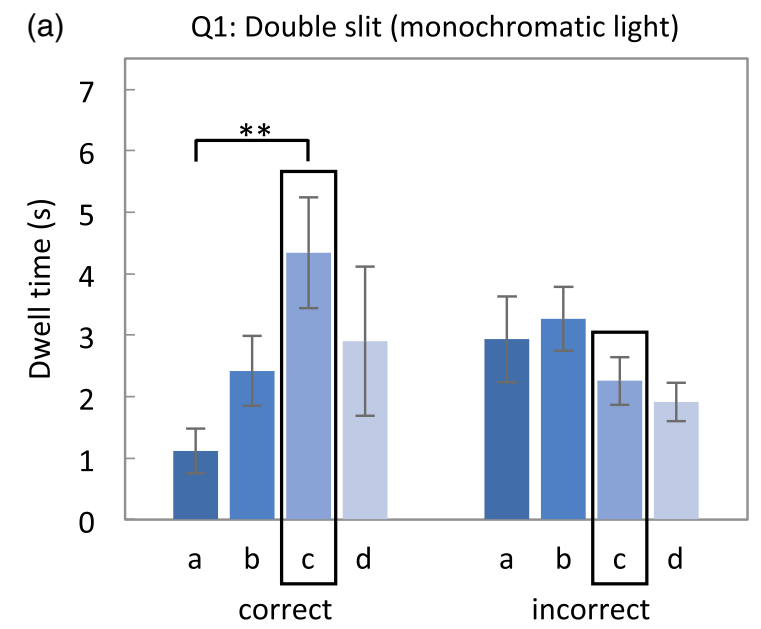

(c) Q3: Diffraction grating (monochromatic light)

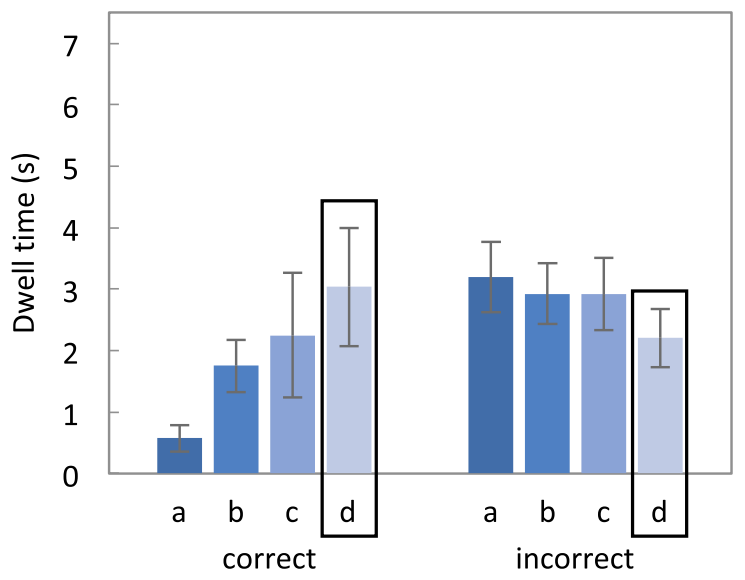

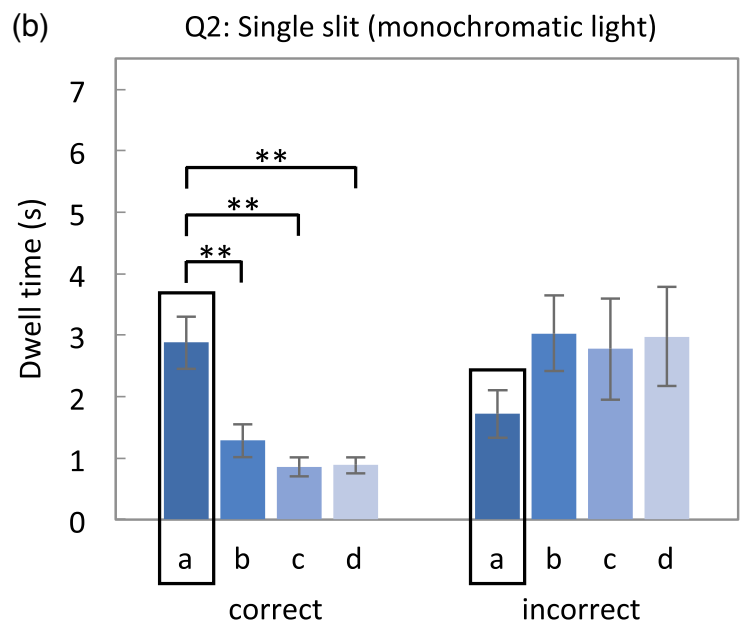

(d) Q4: Diffraction grating (white light)

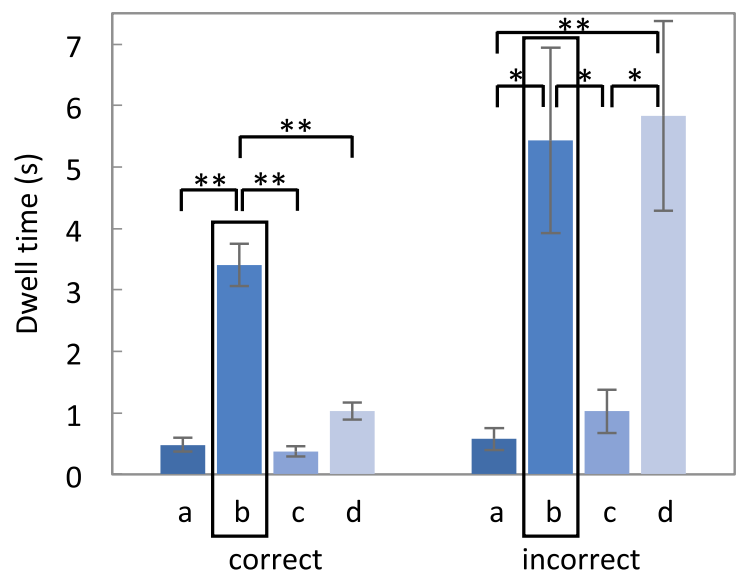

FIG. 2. Dwell times for multiple-choice answers $(a, b, c$, and $d)$ for each question (Q1, Q2, Q3, and Q4), evaluated separately for students who correctly and incorrectly answered the question. Rectangles show correct answers. One or two asterisks indicate significantly different pairwise comparisons at levels of $p<0.05$ or $p<0.01$, respectively.

Students who correctly answered question Q3 had shorter fixation duration for answer $a$ than for answers $b$ and $d$ whereas students who incorrectly answered had longer fixation duration for answer $b$ than for answers $c$ and $d$. For question Q4, students who correctly answered the question had longer fixation durations for the colorful options ( $b$ and $d$ ) compared to gray options ( $a$ and $c$ ). Students who incorrectly answered question Q4 had longer fixation duration for answer $d$ than answer $a$.
Finally, we evaluated and compared the number of fixations and the number of revisits for students who correctly and incorrectly identified interference and diffraction patterns. The results are shown in Figs. S2 and S3, and Tables S2 and S3 in the Supplemental Material [32]. Figures 2 and S2 of Ref. [32] are very similar, which implies that dwell time and the number of fixations are basically highly related eye-tracking measures. The results for the number of revisits (Fig. S3 [32]) are also comparable

TABLE II. Results of the one-way ANOVAs conducted on dwell times for multiple-choice answers $(a, b, c$, and $d)$ for each question, separately for students who correctly and incorrectly answered the question.

\begin{tabular}{lccccccc}
\hline \hline & \multicolumn{3}{c}{ Correct } & & \multicolumn{3}{c}{ Incorrect } \\
\cline { 2 - 3 } & $F(d f)$ & $p$ & $\eta_{p}^{2}$ & & $F(d f)$ & $p$ & $\eta_{p}^{2}$ \\
\hline Q1 double slit & $7.35(3,18)$ & 0.002 & 0.55 & & $2.36(3,81)$ & $>0.05$ & 0.80 \\
Q2 single slit & $23.04(3,63)$ & $<10^{-4}$ & 0.52 & & $1.79(3,36)$ & $>0.05$ & 0.13 \\
Q3 diffraction grating & $2.82(3,18)$ & $>0.05$ & 0.32 & & $1.43(3,81)$ & $>0.05$ & 0.50 \\
Q4 diffraction grating (white light) & $62.32(3,84)$ & $<10^{-4}$ & 0.69 & $8.31(3,15)$ & 0.002 & 0.62 \\
\hline \hline
\end{tabular}



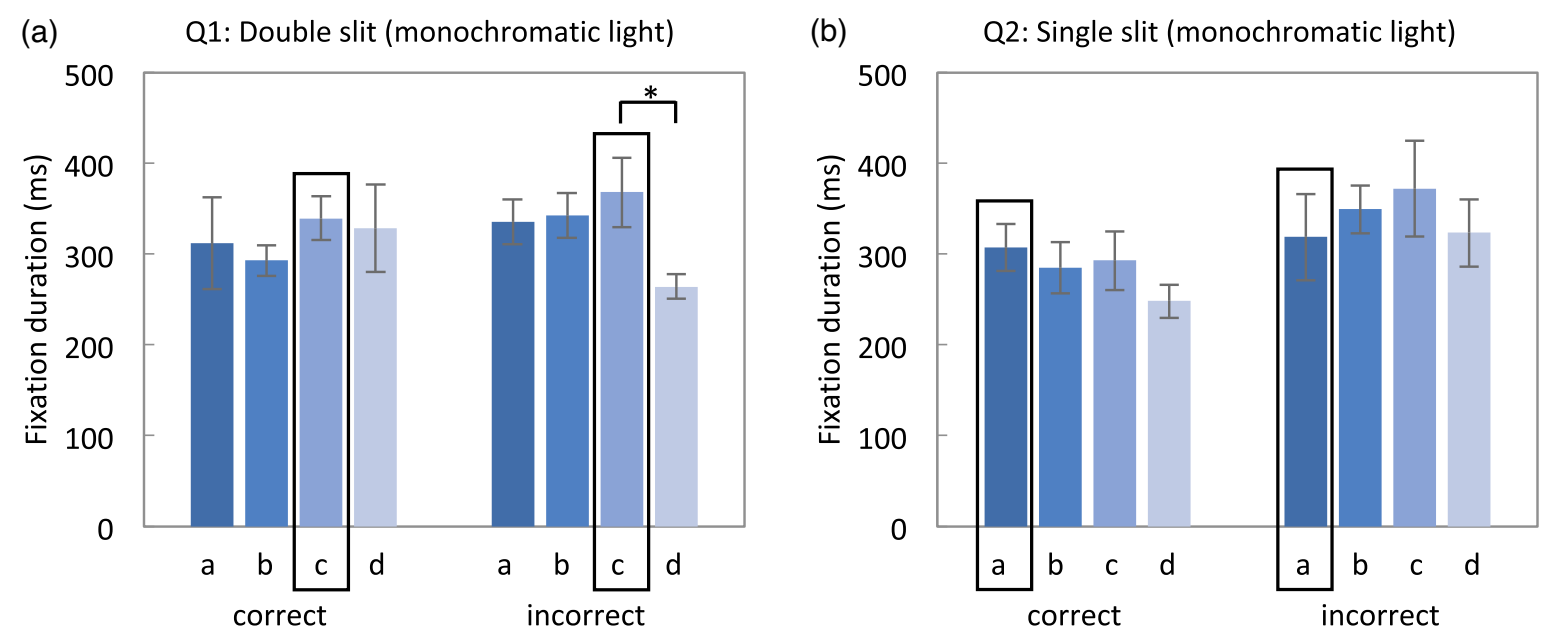

(c) Q3: Diffraction grating (monochromatic light)
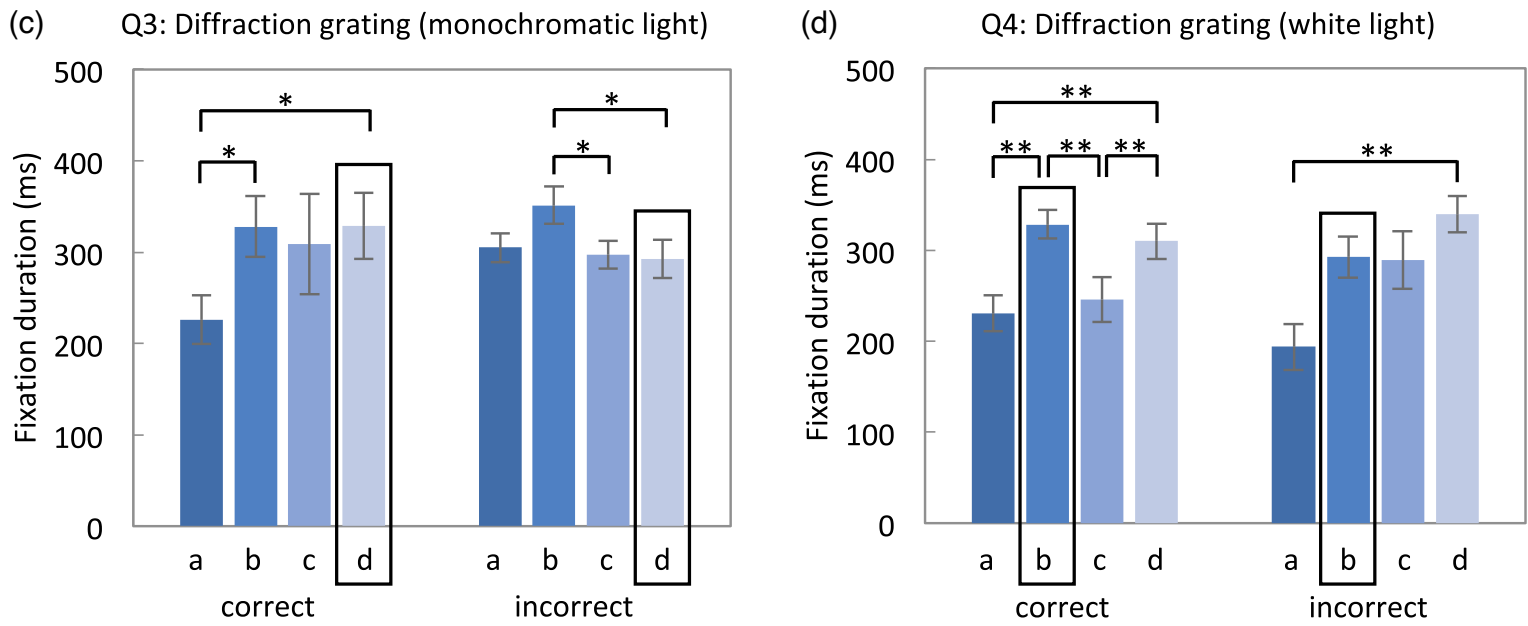

FIG. 3. Fixation durations for multiple-choice answers $(a, b, c$, and $d)$ for each question (Q1, Q2, Q3, and Q4), evaluated separately for students who correctly and incorrectly answered the question. Rectangles show correct answers. One or two asterisks indicate significantly different pairwise comparisons at levels of $p<0.05$ or $p<0.01$, respectively.

to the results for dwell times that again indicates the dependence of these two measures.

\section{DISCUSSION AND CONCLUSION}

Most students had difficulties with identifying the interference and diffraction patterns of monochromatic light used in this study. In particular, students could not recognize the double-slit interference pattern and diffraction grating pattern of monochromatic light. However, they more often identified the single-slit diffraction pattern correctly, which may have been easier to recognize because of its distinguishable central maximum. It might be hypothesized that students confound double-slit interference pattern and diffraction grating pattern because they both have fringes of comparable intensity at approximately equal distance from each other, or that students might not be aware that maxima of diffraction grating pattern are very narrow and separated by relatively wide dark regions.

TABLE III. Results of the one-way ANOVAs conducted on dwell times for multiple-choice answers ( $a, b, c$, and d) for each question, separately for students who correctly and incorrectly answered the question.

\begin{tabular}{lccccccc}
\hline \hline & \multicolumn{3}{c}{ Correct } & & \multicolumn{3}{c}{ Incorrect } \\
\cline { 2 - 3 } & $F(d f)$ & $p$ & $\eta_{p}^{2}$ & & $F(d f)$ & $p$ & $\eta_{p}^{2}$ \\
\hline Q1 double slit & $0.74(3,18)$ & $>0.05$ & 0.55 & & $3.32(3,81)$ & 0.02 & 0.80 \\
Q2 single slit & $0.68(3,63)$ & $>0.05$ & 0.52 & & $0.44(3,36)$ & $>0.05$ & 0.13 \\
Q3 diffraction grating & $4.97(3,18)$ & 0.01 & 0.32 & & $3.80(3,81)$ & 0.01 & 0.50 \\
Q4 diffraction grating (white light) & $12.80(3,84)$ & $<10^{-4}$ & 0.69 & & $5.20(3,15)$ & 0.01 & 0.62 \\
\hline \hline
\end{tabular}


However, the distributions of students' responses for questions Q1 and Q3 suggest that they did not choose only between the double-slit interference pattern and diffraction grating pattern (options $\mathrm{c}$ and $\mathrm{d}$ on the left side of Fig. 1). Moreover, students most frequently chose singleslit interference pattern as an incorrect answer on those questions (option a on the left side of Fig. 1). This suggests that some high-school students tested in our study confused the interference and diffraction patterns of monochromatic light that we used as research materials.

On the other hand, our participants have shown much better recognition of the diffraction pattern of white light on an optical grating. Even $83 \%$ of high-school students answered this question (Q4) correctly, 11\% (4 students) chose option d (colorful stripes) and 6\% (2 students) chose option d (gray stripes). These behavioral results suggest that they knew that diffraction grating separates white light into colors. This was a rather positive result.

Furthermore, eye tracking results revealed that students who identified the tested patterns correctly attended more the correct pattern than other options. This finding is in agreement with the previous studies $[18,24,25]$ which also reported that students who solved a task incorrectly spent the least amount of time on the correct option. Our data showed a similar trend in questions Q2 and Q3, but the differences were not statistically significant. Generally, the distribution of dwell time for multiple-choice answers of students who incorrectly answered the question Q1-Q3 did not show any statistically significant differences. The number of students who chose each particular distractor was rather low, so we did not further divide them into groups according to their (incorrect) answers. However, the distribution of dwell times for multiple-choice answers of students who incorrectly answered the question Q4 showed that they mostly attended the colored patterns. Typical interference and diffraction patterns used in this study are a novel type of stimuli in PER eye-tracking studies and their salience might have an important role in the distribution of visual attention.

Evaluation and comparison of other eye-tracking measures, such as fixation duration, fixation number, and the number of revisits, mostly confirmed dwell time results. Fixation durations were rather similar across different multiple-choice answers, especially for questions Q1 and Q2. Since fixation durations are typically associated with variability in cognitive processing demands, the similarity in fixation durations for all answer options indicated that participants were engaging in the similar, or the same, processes for the selected and not-selected answer options. On the other hand, students who correctly answered question Q3 had the shortest fixation durations for the multiple-choice answer $a$ (the single-slit pattern), which might indicate that they did not have to engage in much cognitive processing to disregard that option. Correspondingly, all students had the shortest fixation durations for option $a$ in question Q4. Students who correctly answered question Q4 had longer fixation duration for the colorful options $b$ and $d$ than the gray options $a$ and $c$. This might imply that these students knew that the pattern should be colorful and could easily disregard grey options. Overall, fixation duration results are consistent to some extent with the dwell time results. On the other hand, the fixation number and the number of revisits are much more congruent with the dwell time results. This corroborates our previous results that these eye-tracking measures are dependent, with fixation duration being rather constant across different tasks whereas dwell time and fixation number show a similar pattern of responses [27].

The distribution of visual attention on question Q4 raises an important question about the nature of visual attention, whether it is a top-down or a bottom-up process. If bottomup processing were crucial in guiding visual attention, we would find visual attention of all participants divided between the two colorful options which are more visually salient than the two gray options. However, this was not the case. As Fig. 2(d) indicates, most participants (83\%) who correctly solved question Q4 spent most of the time attending the correct option $(b)$. The dwell times for the remaining three options (including another colorful option d) were not statistically different from each other. This result corroborates previous findings that students who answer correctly spend most of the time attending the correct option $[15,18,24,25,28]$. Since different multiplechoice options were presented in the previous studies (text, graphs, diagrams, images), it seems that eye movements of participants responding to the multiple-choice questions have a common feature that they attend most the option they choose as correct, and this does seem to depend on perceptual complexity of the options. The result that most participants mainly attended the correct option suggests that top-down processing has an important role in guiding visual attention. The remaining $17 \%$ of the participants, who incorrectly solved question Q4, mostly divided their visual attention between the two colorful options. This could suggest that some students knew that the diffraction of white light on an optical grating produced a colorful pattern, but they did not know other characteristics of the required pattern, so they were indecisive between options $b$ and $d$. It is also possible that some students spent more time attending colorful patterns because of their perceptual salience. Moreover, our results indicate that bottom-up processing could also be important in recognition of wave optics patterns (e.g., colored patterns are salient, capture more attention and students better memorize them). This should be explored in more details in future studies.

Furthermore, the analysis of students' responses and eyetracking data validated the design of the multiple-choice questions used in this study. Questions Q1-Q3 used the same multiple-choice options, so choosing one option on question Q1 might have decreased the probability of 
choosing that answer in the subsequent questions. One could expect that the students would distribute their answers on questions Q1-Q3 relatively uniformly, given that the options were always the same. However, the results in Table I indicate that was not the case; in all three questions, their preferred answer was $a$ (the single-slit pattern). Moreover, the same position of multiple-choice patterns in questions Q1-Q3 might have influenced the eye movements. The inspection of students' eye movements did not confirm that they remembered the options from the previous questions and only had to choose the correct answer. They almost always checked all four answers, which is also evident from their dwell times in Fig. 2. It is possible that their first inspection of the answers was longer the first time when they encountered them. However, the questions Q1-Q3 were presented in counterbalanced order, so it did not have a confounding effect overall.

In this study, the participants were high-school students. We assume that similar results would be obtained for firstyear university students. They are only one year older than participants in our study and they cover similar topics in their curriculum. At the university level, some aspects of interference and diffraction of light are introduced in a more formal way compared to high school. However, we believe that difficulties with the basic concepts of wave optics, and with recognition and identification of typical interference and diffraction patterns would be found for students in introductory university physics courses; only the prevalence of certain difficulties would probably not be the same as for high-school students. Some previous studies, using instruments such as the Force Concept Inventory, have shown that university students have similar difficulties with conceptual understanding in mechanics as high-school students, e.g., Ref. [33]. However, additional studies are needed to confirm that the first-year university students show similar difficulties in distinguishing typical wave optics patterns as high-school students.
In summary, this study showed that high-school students had difficulties recognizing some typical interference and diffraction patterns. This task is probably more complex than it may seem because many students were not familiar with these patterns from their own experimental experience, as they only had a chance to see these patterns in their textbooks or demonstration experiments performed by teachers. Also, some patterns may be visually very similar from the student point of view, which may present an obstacle to their differentiation by the students and to students' learning [34]. Students may need more time and guidance in hands-on experiments with those patterns to compare and contrast them and really notice and remember their key features and differences. However, although some aspects of physical concepts (such as interference or diffraction) can be represented through experiments, mathematical representation is also required to characterize other aspects of those concepts [35]. Application of multiple representations is important in developing a deeper understanding of physical concepts. In future studies, we will investigate whether students' recognition of the interference and diffraction patterns can be improved by students' taking part in inquiry-based activities including experiments performed by students. We expect that such experimental activities could increase students' ability to recognize typical interference and diffraction patterns, as well as motivate them to engage further in searching for their explanations. The results of the present study indicate that this first step already is, at least in part, difficult for high-school students, so it can be concluded that it needs more attention in the teaching process.

\section{ACKNOWLEDGMENTS}

This work has been supported in part by the Croatian Science Foundation under the Project No. IP-201801-9085.
[1] B. S. Ambrose, P. S. Shaffer, R. N. Steinberg, and L. C. McDermott, An investigation of student understanding of single-slit diffraction and double-slit interference, Am. J. Phys. 67, 146 (1999).

[2] K. Wosilait, P. R. L. Heron, P. S. Shaffer, and L. C. McDermott, Addressing student difficulties in applying a wave model to the interference and diffraction of light, Am. J. Phys. 67, S5 (1999).

[3] P. Colin and L. Viennot, Using two models in optics: Students' difficulties and suggestions for teaching, Am. J. Phys. 69, S36 (2001).

[4] L. Maurines, Geometrical reasoning in wave situations: The case of light diffraction and coherent illumination optical imaging, Int. J. Sci. Educ. 32, 1895 (2010).
[5] A. Coetzee and S. N. Imenda, Alternative conceptions held by first year physics students at a South African university of technology concerning interference and diffraction of waves, Res. High. Educ. J. 16, 13 (2012), http://www.aabri .com/manuscripts/121097.pdf.

[6] V. Mešić, K. Neumann, I. Aviani, E. Hasović, W. J. Boone, N. Erceg, V. Grubelnik, A. Sušac, D. S. Glamočić, M. Karuza, A. Vidak, A. Alihodžić, and R. Repnik, Measuring students' conceptual understanding of wave optics: A Rasch modeling approach, Phys. Rev. Phys. Educ. Res. 15, 010115 (2019).

[7] V. Mešić, A. Vidak, E. Hasović, and M. Dekić, University students' ideas about the role of the aperture and laser beam 
dimensions in formation of diffraction patterns, Eur. J. Phys. 40, 055701 (2019).

[8] R. Dai, J. C. Fritchman, Q. Liu, Y. Xiao, H. Yu, and L. Bao, Assessment of student understanding on light interference, Phys. Rev. Phys. Educ. Res. 15, 020134 (2019).

[9] P. Hubber, Year 12 students' mental models of the nature of light, Res. Sci. Educ. 36, 419 (2006).

[10] V. Mešić, E. Hajder, K. Neumann, and N. Erceg, Comparing different approaches to visualizing light waves: An experimental study on teaching wave optics, Phys. Rev. Phys. Educ. Res. 12, 010135 (2016).

[11] S. K. Şengören, How do Turkish high school graduates use the wave theory of light to explain optics phenomena?, Phys. Educ. 45, 253 (2010).

[12] M. Kryjevskaia, M. R. Stetzer, and P. R. L. Heron, Student understanding of wave behavior at a boundary: The relationships among wavelength, propagation speed, and frequency, Am. J. Phys. 80, 339 (2012).

[13] M. Kryjevskaia, M. R. Stetzer, and P. R. L. Heron, Student difficulties measuring distances in terms of wavelength: Lack of basic skills or failure to transfer?, Phys. Rev. ST Phys. Educ. Res. 9, 010106 (2013).

[14] A. D. Smith, J. P. Mestre, and B. H. Ross, Eye-gaze patterns as students study worked-out examples in mechanics, Phys. Rev. ST Phys. Educ. Res. 6, 020118 (2010).

[15] A. M. Madsen, A. M. Larson, L. C. Loschky, and N. S. Rebello, Differences in visual attention between those who correctly and incorrectly answer physics problems, Phys. Rev. ST Phys. Educ. Res. 8, 010122 (2012).

[16] A. Susac, A. Bubic, E. Kazotti, M. Planinic, and M. Palmovic, Student understanding of graph slope and area under a graph: A comparison of physics and nonphysics students, Phys. Rev. Phys. Educ. Res. 14, 020109 (2018).

[17] P. Klein, S. Küchemann, S. Brückner, O. Zlatkin-Troitschanskaia, and J. Kuhn, Student understanding of graph slope and area under a curve: A replication study comparing first-year physics and economics students, Phys. Rev. Phys. Educ. Res. 15, 020116 (2019).

[18] P. Klein, A. Lichtenberger, S. Kuchemann, S. Becker, M. Kekule, J. Viiri, C. Baadte, A. Vaterlaus, and J. Kuhn, Visual attention while solving the test of understanding graphs in kinematics: An eye-tracking analysis, Eur. J. Phys. 41, 025701 (2020).

[19] P. Klein, J. Viiri, S. Mozaffari, A. Dengel, and J. Kuhn, Instruction-based clinical eye-tracking study on the visual interpretation of divergence: How do students look at vector field plots?, Phys. Rev. Phys. Educ. Res. 14, 010116 (2018).

[20] A. Madsen, A. Rouinfar, A. M. Larson, L. C. Loschky, and N.S. Rebello, Can short duration visual cues influence students' reasoning and eye movements in physics problems?, Phys. Rev. ST Phys. Educ. Res. 9, 020104 (2013).

[21] A. Rouinfar, E. Agra, A. M. Larson, N. S. Rebello, and L. C. Loschky, Linking attentional processes and conceptual problem solving: Visual cues facilitate the automaticity of extracting relevant information from diagrams, Front. Psychol. 5, 1094 (2014).

[22] P. Klein, J. Viiri, and J. Kuhn, Visual cues improve students' understanding of divergence and curl: Evidence from eye movements during reading and problem solving, Phys. Rev. Phys. Educ. Res. 15, 010126 (2019).

[23] S. C. Chen, H. C. She, M. H. Chuang, J. Y. Wu, J. L. Tsai, and T. P. Jung, Eye movements predict students' computerbased assessment performance of physics concepts in different presentation modalities, Comput. Educ. 74, 61 (2014).

[24] J. Han, L. Chen, Z. Fu, J. Fritchman, and L. Bao, Eyetracking of visual attention in web-based assessment using the Force Concept Inventory, Eur. J. Phys. 38, 045702 (2017).

[25] M. Kekule and J. Viiri, Students' approaches to solving R-FCI tasks observed by eye-tracking method, Sci. Educ. 9, 117 (2018).

[26] A. Susac, A. Bubic, P. Martinjak, M. Planinic, and M. Palmovic, Graphical representations of data improve student understanding of measurement and uncertainty: An eye-tracking study, Phys. Rev. Phys. Educ. Res. 13, 020125 (2017).

[27] A. Susac, A. Bubic, M. Planinic, M. Movre, and M. Palmovic, Role of diagrams in problem solving: An evaluation of eye-tracking parameters as a measure of visual attention, Phys. Rev. Phys. Educ. Res. 15, 013101 (2019).

[28] M. J. Tsai, H. T. Hou, M. L. Lai, W. Y. Liu, and F. Y. Yang, Visual attention for solving multiple-choice science problem: An eye-tracking analysis, Comput. Educ. 58, 375 (2012).

[29] https://www.itp.uni-hannover.de/fileadmin/arbeitsgruppen/ zawischa/static_html/multibeam.html.

[30] K. J. Rothman, No adjustments are needed for multiple comparisons, Epidemiology 1, 43 (1990).

[31] A. Gelman, J. Hill, and M. Yajima, Why We (Usually) Don't Have to Worry About Multiple Comparisons, J. Res. Educ. Eff. 5, 189 (2012).

[32] See Supplemental Material at http://link.aps.org/ supplemental/10.1103/PhysRevPhysEducRes.16.020133 contains comparisons of proportions of dwell time, fixation numbers and revisits for students who correctly and incorrectly answered questions.

[33] M. Planinic, L. Ivanjek, and A. Susac, Rasch model based analysis of the Force Concept Inventory, Phys. Rev. ST Phys. Educ. Res. 6, 010103 (2010).

[34] W. M. Roth, C. J. McRobbie, K. B. Lucas, and S. Boutonné, Why may students fail to learn from demonstrations? A social practice perspective on learning in physics, J. Res. Sci. Teach. 34, 509 (1997).

[35] J. Airy and C. Linder, Social semiotics in university physics education, in Multiple Representations in Physics Education, edited by D. Treagust, R. Duit, and H. Fischer (Springer, Cham, 2017), pp. 95-122. 